\title{
Laser-Written Silicon-Germanium Alloy Microstructures with Tunable Compositionally Graded Profiles
}

\author{
Ozan Aktas $^{1, *}$, Stuart J. MacFarquhar ${ }^{1}$, Swe Z. Oo ${ }^{1,2}$, Vinita Mittal ${ }^{1}$, Harold M. H. Chong ${ }^{2,3}$, and \\ Anna C. Peacock ${ }^{1}$ \\ ${ }^{1}$ Optoelectronics Research Centre, University of Southampton, Southampton, SO17 1BJ, UK \\ ${ }^{2}$ School of Electronics and Computer Science, University of Southampton, Southampton, SO17 1BJ, UK \\ ${ }^{3}$ School of Materials Science, Japan Advanced Institute of Science and Technology, Ishikawa, 923-1292, Japan \\ *O.Aktas@soton.ac.uk
}

\begin{abstract}
A laser processing method is introduced for post-deposition tailoring of local composition and bandgap in amorphous silicon-germanium thin films on silicon substrates. Spatial distribution of the alloy constituents can be controlled through the scan speed. (c) 2020 The Author(s)
\end{abstract}

\section{Introduction}

An emerging application of lasers is to gain control over the phase segregation or transformation in multicomponent solids for microscale manipulation of the material properties [1]. Local modification of the structure and composition within silicon-germanium (SiGe) core glass-clad fibers has been recently achieved [2]. However, tailoring of the local composition in semiconductor alloy films on planar substrates using lasers has yet to be demonstrated. Here we report post-deposition tailoring of the local composition in amorphous SiGe nanoscale thin films on silicon substrates by controlling the phase segregation of the SiGe through the laser scan speed. Our approach allows for fabrication of spatially graded $\mathrm{Si}_{1-x} \mathrm{Ge}_{x}$ microstripes almost covering the entire compositional range $(0<x<1)$. As an application, we demonstrate fabrication of photoconductive detectors by exploiting the laserwritten polycrystalline (poly-SiGe) microstripes. Their bandgap edges are tunable over a $200 \mathrm{~nm}$ range by changing the laser scan speed during processing, due to the scan-speed-dependent Ge redistribution in the solidified region.

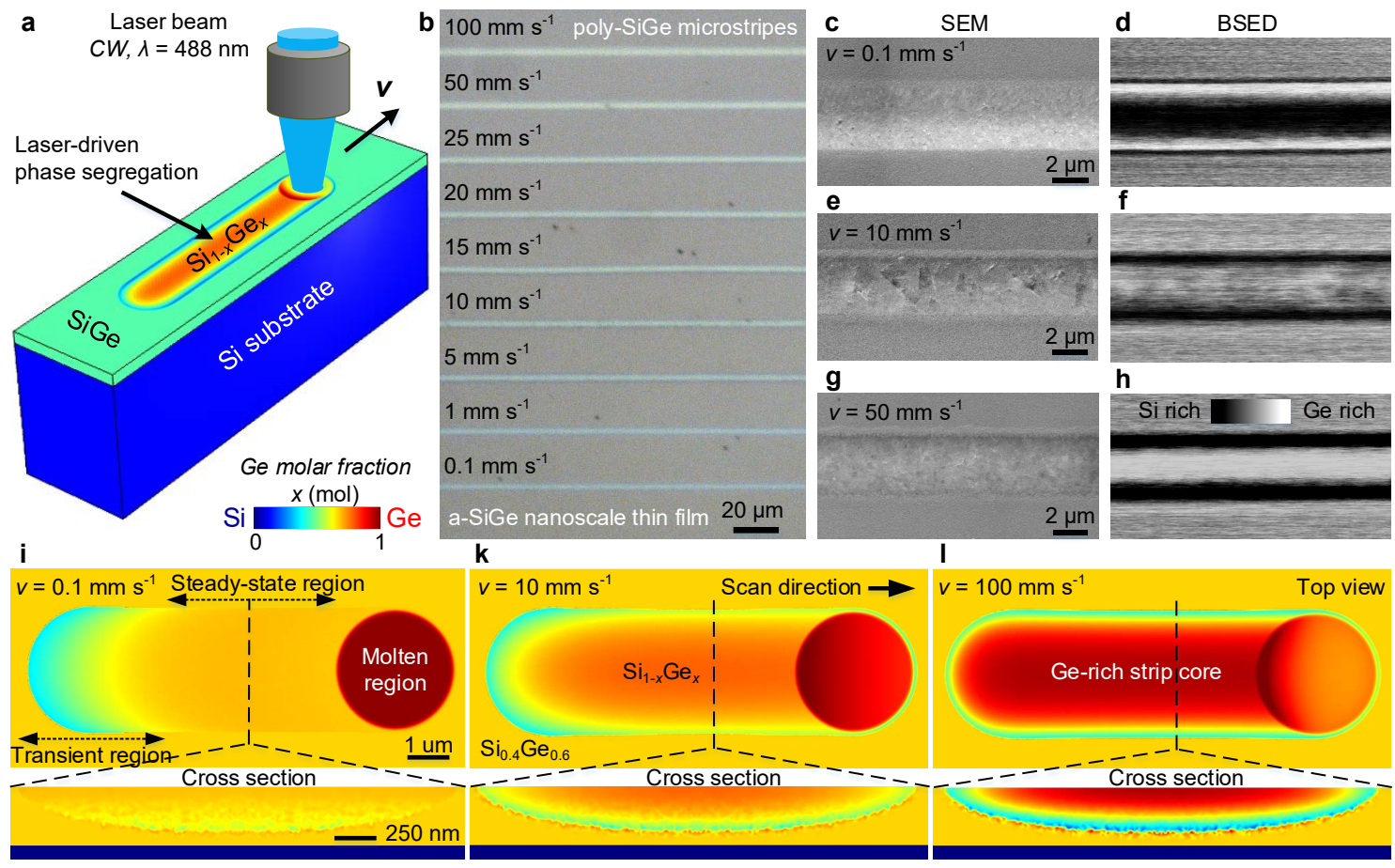

Fig. 1. a) Schematic of laser writing process. b) Optical image of $3 \mu \mathrm{m}$ wide laser-written poly-SiGe microstripes showing a color shading from blue to yellow depending on the Ge composition on the top surface. c-h) Scanning electron microscopy (SEM) and back-scattered electron detector (BSED) micrographs show the structural and compositional contrast between Ge-rich (bright) and Si-rich (dark) regions, as given for scan speeds of (c,d) $0.1 \mathrm{~mm} \mathrm{~s}^{-1}$, (e,f) $10 \mathrm{~mm} \mathrm{~s}^{-1}$, and (g,h) $50 \mathrm{~mm} \mathrm{~s}^{-1}$, respectively. i-l) Phase-field simulations of laser-driven phase segregation. Color maps show the distribution of the $\mathrm{Si}_{1-x} \mathrm{Ge}_{x}$ composition after laser processing at scan speeds of (i) 0.1 , (k) 10 , and (l) $100 \mathrm{~mm} \mathrm{~s}$. https://pubs.acs.org/doi/abs/10.1021/acsami.9b22135 


\section{Direct laser writing of polycrystalline SiGe (poly-SiGe) microstripes}

Laser writing was undertaken with a CW Argon-ion laser source at a wavelength of $488 \mathrm{~nm}$. The beam was focused on the top surface of the $400 \mathrm{~nm}$ thick a-SiGe film (deposited by PECVD) by using a 20X lens to produce a spot size of diameter $3 \mu \mathrm{m}$. Samples were mounted on motorized stages and then scanned under the laser beam at speeds ranging from 0.1 to $100 \mathrm{~mm} \mathrm{~s}^{-1}$ (Fig. 1a,b). The power was adjusted between 200-320 mW. The experimental data reveals the formation of a Ge-rich strip core, surrounded by a Si-rich under-cladding in the laser-melted volume, where the spatial distribution of the alloy constituents is directly determined by the laser scan speed (see Fig. 1c-h).

\section{Finite-element-method-based phase-field simulations of laser-driven phase segregation}

We have extensively investigated the laser-driven phase segregation, by using FEM-based phase-field simulations in 3D, which are in excellent agreement with the experimental results. Figure 1i-l shows the spatial distribution of the Ge molar fraction $x(\boldsymbol{r}, t)$ after laser writing at different speeds. Initial overall Ge molar fraction is $x_{0}=0.6$ mol. The laser-induced molten zone (red color) travels along the scan direction dragging the Ge-rich liquid, which solidifies at the trailing edge. After a Si-rich initial transient (cyan color), a steady-state region emerges with a Ge-rich strip core and a Si-rich under-cladding. Complete mixing occurs in the molten zone for low scan speeds. However, the Ge content $x$ builds up at the trailing edge (darker red), due to insufficient diffusion-limited Ge transport at high speeds.

\section{Characterization of material and optoelectronic properties of laser-written poly-SiGe microstripes}

Characterization of the laser-melted poly- $\mathrm{Si}_{1-x} \mathrm{Ge}_{x}$ regions was achieved using micro-focus synchrotron X-ray diffraction (XRD), Raman spectroscopy and scanning electron microscopy (SEM) with a back-scattered electron detector (BSED). 1D X-ray diffractograms of the laser-written poly-SiGe microstripes are given in Fig. 2a. A pulsed broadband supercontinuum laser with an acousto-optic tunable filter was then used as an excitation source for I-V and spectral responsivity measurements. We tested a series of surface-normal-illuminated photoconductive detectors with metal-semiconductor-metal contacts, by placing two stainless steel metal probes onto the surface of the laserwritten poly-SiGe microstripes. The spectral onset of the measurable photocurrent progressively shifts over a range of $200 \mathrm{~nm}$, with the accumulation of Ge on the top surface associated with the faster scan speeds (see Fig. 2b).
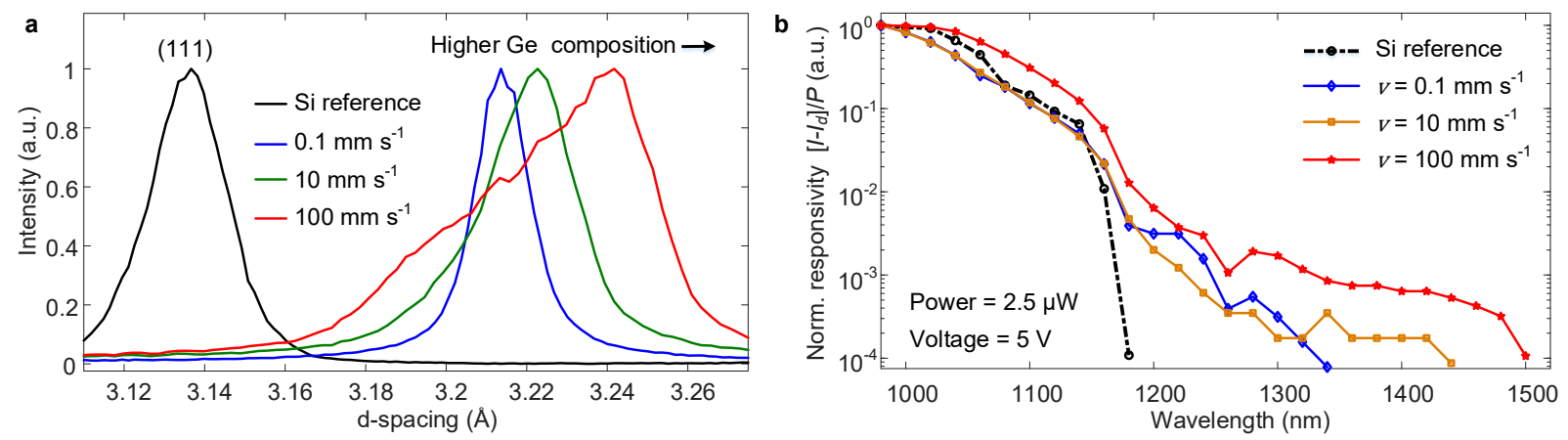

Fig. 2. a) X-ray diffractograms of the laser-written poly-SiGe microstripes showing peak broadening and shifting for higher scan speeds, which can be deconvolved into Ge-rich and Si-rich peaks. b) Normalized spectral responsivity of the photodetectors produced on the laserwritten poly-SiGe microstripes with various composition profiles induced by phase segregation driven at different constant scan speeds.

\section{Conclusion}

Using the laser-driven phase segregation, we produced indefinitely long poly-SiGe microstructures, where the crosssectional composition profiles are determined by setting the laser scan speed during laser processing. On-chip, postdeposition laser tailoring of the composition can also be applied to epitaxially grown SiGe films, and pseudo-binary alloys of ternary semiconductors, metals, ceramics and organic crystals, which have phase diagrams similar to that of SiGe alloys. This study paves the way for direct laser writing of novel devices made of alloy microstructures with tunable composition profiles, including graded-index waveguides and meta-surfaces, multispectral photodetectors, full-spectrum solar cells, and lateral heterostructures. The authors thank Prof. Sakellaris Mailis and Dr. Konstantin Ignatyev, and acknowledge the use of beamline I18 at the Diamond Light Source and EPSRC for funding.

\section{References}

[1] I. Choi, H. Y. Jeong, H. Shin, G. Kang, M. Byun, H. Kim, A. M. Chitu, J. S. Im, R. S. Ruoff, S.-Y. Choi, and K. J. Lee, "Laser-induced phase separation of silicon carbide,“ Nat. Commun. 7, 13562 (2016).

[2] D. A. Coucheron, M. Fokine, N. Patil, D. W. Breiby, O. T. Buset, N. Healy, A. C. Peacock, T. Hawkins, M. Jones, J. Ballato, U. J. Gibson,

"Laser recrystallization and inscription of compositional microstructures in crystalline SiGe-core fibres," Nat. Commun. 7, 13265 (2016). 